\title{
Gambogic Acid, a Natural Product Inhibitor of Hsp90
}

\author{
Jason Davenport, ${ }^{\dagger}$ Jacob R. Manjarrez, ${ }^{\dagger}$ Laura Peterson, ${ }^{\ddagger}$ Brian Krumm, ${ }^{\dagger}$ Brian S. J. Blagg, and \\ Robert L. Matts, \\ ${ }^{\dagger}$ Department of Biochemistry and Molecular Biology, 246 Noble Research Center, Oklahoma State University, Stillwater, \\ Oklahoma 74078, United States \\ ${ }^{\ddagger}$ Department of Medicinal Chemistry, 1251 Wescoe Hall Drive, Malott 4070, The University of Kansas, Lawrence, Kansas 66045, United States
}

ABSTRACT: A high-throughput screening of natural product libraries identified (-)-gambogic acid (1), a component of the exudate of Garcinia harburyi, as a potential Hsp90 inhibitor, in addition to the known Hsp90 inhibitor celastrol (2). Subsequent testing established that 1 inhibited cell proliferation, brought about the degradation of $\mathrm{Hsp} 90$ client proteins in cultured cells, and induced the expression of Hsp70 and Hsp90, which are hallmarks of Hsp90 inhibition. Gambogic acid also disrupted the interaction of Hsp90, Hsp70, and Cdc37 with the heme-regulated eIF2 $\alpha$ kinase (HRI, an Hsp90-dependent client) and blocked the maturation of HRI in vitro. Surface plasmon resonance spectroscopy indicated that $\mathbf{1}$ bound to the $\mathrm{N}$-terminal domain of $\mathrm{Hsp} 90$ with a low micromolar $K_{\mathrm{d}}$, in a manner that was not competitive with the Hsp90 inhibitor geldanamycin (3). Molecular docking experiments supported the posit that $\mathbf{1}$ binds Hsp90 at a site distinct from Hsp90s ATP binding pocket. The data obtained have firmly established $\mathbf{1}$ as a novel Hsp90 inhibitor and have provided evidence of a new site that can be targeted for the development of improved Hsp90 inhibitors.

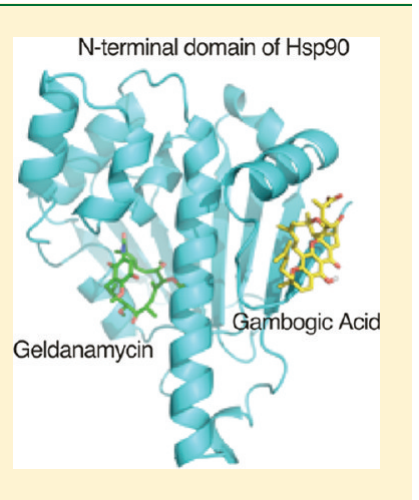

$\mathrm{T}$ he $90 \mathrm{kDa}$ heat shock protein (Hsp90) is the core component of an oligomeric chaperone machine, the function of which is required for the viability of all eukaryotic cells. Hsp90 functions with a cohort of co-chaperones to facilitate the folding, activation, and stabilization of numerous client proteins, many of which have effects in regulating signal transduction pathways. ${ }^{1,2}$ Among the plethora of Hsp90-dependent clients are proteins that function in pathways that represent all six hallmarks of cancer. ${ }^{3-6}$ Thus, inhibition of Hsp90 function simultaneously incapacitates multiple client proteins, providing a combinatorial attack on cellular oncogenic processes. Consequently, Hsp90 has emerged as an exciting new target for the development of antitumor agents.

Natural product inhibitors of $\mathrm{Hsp} 90$ have been discovered that target binding sites in the $\mathrm{N}$ - and C-terminal domains of Hsp90. Geldanamycin (3) and radicicol, which are produced by the soil actinomycetes species Streptomyces hygroscopicus and the mycoparasitic fungus Humicola fuscoatra, respectively, bind to the ATP binding pocket in Hsp90s N-terminal domain, ${ }^{1,2}$ while novobiocin (4), which is produced by Streptomyces spheroides, inhibits Hsp90 function by binding to Hsp90s C-terminal domain. $^{7}$ Other natural products with well-known antitumor and/or chemopreventive properties, but poorly characterized mechanisms of action have been discovered to exhibit inhibitory activity toward Hsp90: epigallocatechin gallate (EGCG), ${ }^{8,9}$ the well-known antioxidant found in green tea (Camellia sinensis (L.) Kuntze); gedunin, a tetranortriterpenoid isolated from the Indian neem tree (Azadirachta indica A. Juss.; Meliaceae); ${ }^{10,11}$ celastrol (2), a quinone methide triterpene that is a pharmacologically active compound present in Thunder God Vine root extracts (Tripterygium wilfordii Hook.f.; Celastraceae); ${ }^{11,12}$ and the rotenoid deguelin. ${ }^{13}$
Derivatives of geldanamycin (3) and other compounds that target the ATP-binding pocket in Hsp90s N-terminal domain have entered more than 20 clinical trials for the treatment of cancer. ${ }^{5,14,15}$ Clinical complications have arisen in phase II trials of several Hsp90 inhibitors, with incidences of hepato-, cardio-, and ocular toxicity having dampened enthusiasm for the clinical use of Hsp90 inhibitors. ${ }^{5}$ Consequently, there is an ongoing search for Hsp90 inhibitors with superior chemotherapeutic properties for the treatment of cancers.

To this end, we have screened natural product libraries for compounds that inhibit Hsp90-dependent refolding of thermally denatured firefly luciferase. It was presumed that natural products represent a fertile territory for the identification of new Hsp90-inhibitors, as it is reasonable to expect that evolutionary pressures give plants that produce secondary metabolites inhibitory to Hsp90 a competitive advantage, because such compounds might inhibit the growth and development of insect pests and other pathogens. Celastrol (2), a known Hsp90 inhibitor, ${ }^{1,12}$ and (-)-gambogic acid (1), a component of Garcinia hanburyi Hook.f. (Clusiaceae), a species that has been used medicinally for centuries in southeast Asia, were identified as inhibitors of luciferase refolding in screens of two natural product libraries.

Gambogic acid (1), like Hsp90 inhibitors, has antitumor, antiangiogenic, and antimetastatic activities (reviewed in refs 16-18), but a poorly characterized mechanism of action. In addition, like Hsp90 inhibitors, ${ }^{19} 1$ has been observed to be selectively cytotoxic to cancer versus normal cells. ${ }^{20,21}$ While $\mathbf{1}$

Received: January 10, 2011

Published: April 12, 2011 
has been reported to induce apoptosis in cancer cells by binding to the transferrin receptor, ${ }^{22}$ the cytotoxic activity of this compound has also been found to have a transferrin receptorindependent component. ${ }^{23} \mathrm{~A}$ recent publication on gambogic acid (1) indicates that $\mathbf{1}$ has recently been subjected to a phase I clinical trial in the People's Republic of China as an anticancer agent. ${ }^{24}$ Herein, we present the characterization of the Hsp90 inhibitory activity of $\mathbf{1}$ and compare its mechanism of action to those of other Hsp90 inhibitors.

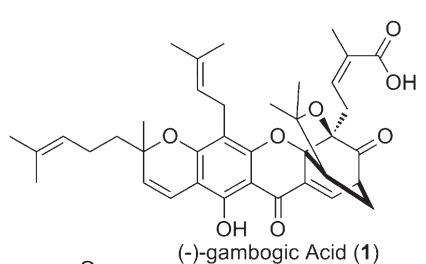

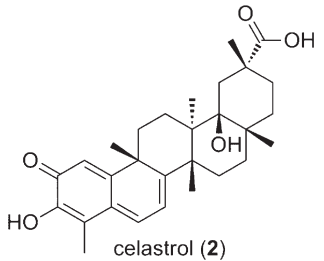

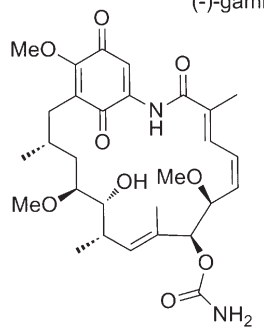
geldanamycin (3)<smiles>CC(C)=CCc1cc(C(=O)Nc2c(O)c3ccc(OC4C(O)C5(C)OC(C)C4(C)O5)c(C)c3oc2=O)ccc1O</smiles>

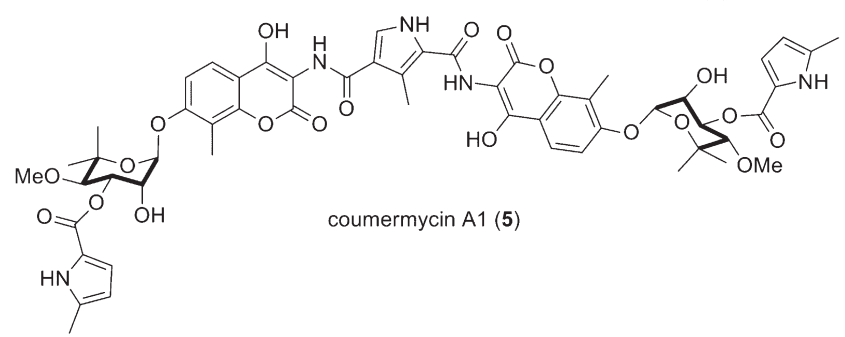

\section{RESULTS AND DISCUSSION}

Identification of Gambogic Acid (1) as a Putative Hsp90 Inhibitor from a High-Throughput Screen of Natural Product Libraries. Screening of natural product libraries purchased from Microsource and Biomol for compounds that inhibited Hsp90-dependent refolding of luciferase identified 1 as a potential Hsp90 inhibitor, along with the known Hsp90 inhibitor celastrol (2), among other compounds. Neither celastrol nor $\mathbf{1}$ had any direct effect on the activity of native luciferase. Upon titration of various concentrations of the two compounds into the refolding assay (Figure 1A), celastrol (2) and gambogic acid (1) were found to inhibit luciferase refolding by $50 \%\left(\mathrm{IC}_{50}\right)$ at a concentration of 20 and $2 \mu \mathrm{M}$, respectively.

Gambogic acid (1) has been demonstrated in numerous studies to inhibit the proliferation of a variety of cancer cell lines (reviewed in refs 16-18). To determine whether antiproliferative activity of 1 could be correlated with its Hsp90-inhibitory activity, we examined the effect of varying concentrations of gambogic acid on the growth/viability of HeLa cells and MCF7 and SK-Br3 breast cancer cell lines. Gambogic acid (1) inhibited the proliferation of HeLa, MCF7, and SK-Br3 cells in a concentration-dependent manner (Figure 1B). Growth of the HeLa, MCF7, and SK-Br3 cells was inhibited by $50 \%$ by treatment with $1.5,2.0$, and $0.8 \mu \mathrm{M} 1$, respectively.
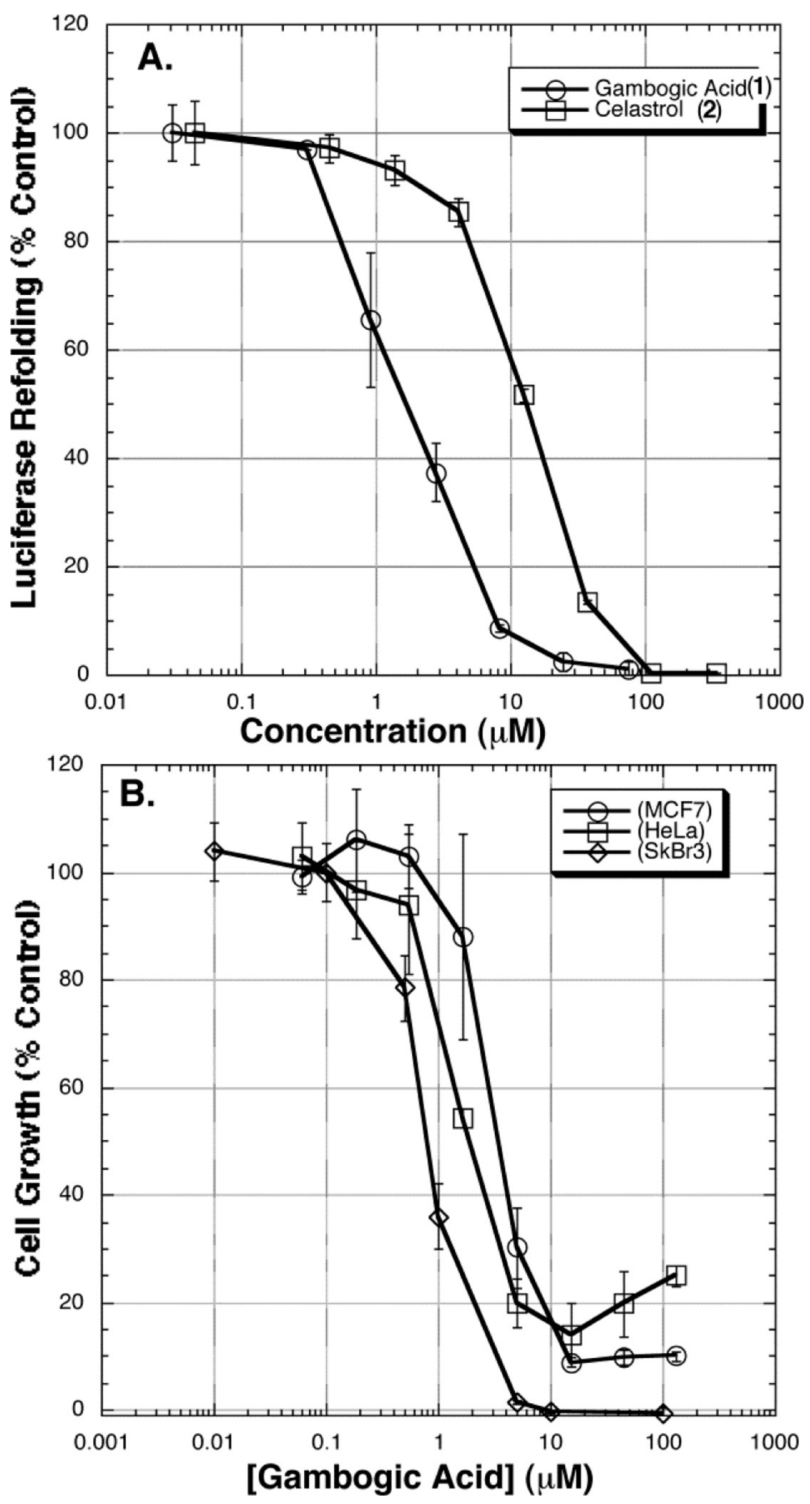

Figure 1. Effect of gambogic acid (1) and celastrol (2) on Hsp90dependent luciferase refolding in reticulocyte lysate (A) and effect of $\mathbf{1}$ on cell proliferation of HeLa cells and MCF7 and SkBr3 breast cancer cells (B). Experiments were carried out as described in the Experimental Section.

The highest concentrations of $\mathbf{1}$ were cytotoxic, as evidenced by detachment of a significant number of cells from the surface of the culture flasks. Thus, the $\mathrm{IC}_{50}$ of $\mathbf{1}$ for inhibition of cell proliferation correlated well with its $\mathrm{IC}_{50}$ for the inhibition of luciferase refolding.

Gambogic Acid (1)-Induced Depletion of Hsp90-Dependent Proteins. Treatment of cultured cells with known Hsp90 inhibitors depletes the cells of Hsp90-dependent proteins in a time- and concentration-dependent manner. To further characterize $\mathbf{1}$ as a potential Hsp90 inhibitor, MCF7 and Sk-Br3 cells were treated with varying concentrations of $\mathbf{1}$ for $36 \mathrm{~h}$, and equivalent amounts of protein from cell extracts were Western blotted for Hsp70 and Hsp90 and the Hsp90-dependent proteins Her2, Akt, and Raf-1, using actin as a loading control, geldanamycin (3) as a positive control for Hsp90 inhibition, and DMSO as a negative control. Gambogic acid was observed to deplete 


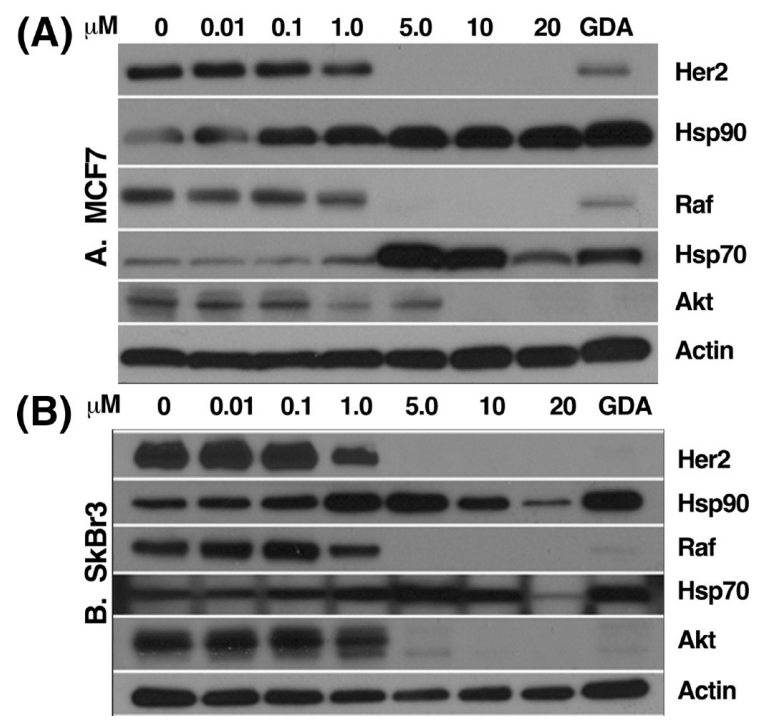

Figure 2. Gambogic acid (1)-induced degradation of Hsp90 client proteins. Compound 1 was incubated with (A) MCF7 and (B) SkBr3 breast cancer cells at concentrations $(\mu \mathrm{M})$ indicated in the figure. Gambogic acid (1) was evaluated for its ability to downregulate several client proteins as described in the Experimental Section. Geldanamycin (3) (500 nM) and DMSO were used as positive and negative controls, respectively. Cell extracts were prepared and equivalent amounts of protein were separated by SDS-PAGE and subsequently Western blotted for the indicated proteins as described in the Experimental Section.

MCF7 and Sk-Br3 cells of Her2, Akt, and Raf-1 in a concentration-dependent fashion (Figure 2), which correlated well with the $\mathrm{IC}_{50}$ value for inhibition of the proliferation of these cell lines induced by 1 . In addition, 1 induced Hsp90 and Hsp70 expression, another hallmark of Hsp90 inhibition. This compound had a similar effect on the levels of Her2, Raf-1, and Akt in HeLa cells (not shown). These results further support the hypothesis that the antiproliferative effect of $\mathbf{1}$ on cancer cell growth is mediated, at least in part, by its ability to inhibit Hsp90.

Effect of Gambogic Acid (1) on the Association of Hsp90 Chaperone Components with HRI and HRI's Hsp90-Dependent Maturation. Hsp90 inhibitors have distinct effects on the conformation and interactions of Hsp90 with co-chaperone partners and clients. ${ }^{25,26}$ Geldanamycin (3), an Hsp90 inhibitor that binds to the ATP pocket in the N-terminal domain of Hsp90 (N-terminal inhibitor), causes Hsp90 to accumulate in complexes that contain intermediate co-chaperone components. Complexes that form between Hsp90 and kinase clients in the presence of 3 are salt-labile and lack Cdc37. Molybdate, on the other hand, inhibits Hsp90 by freezing it in complexes containing late co-chaperone components. Hsp90-kinase client complexes formed in the presence of molybdate are stabile at high salt concentrations (e.g., $0.5 \mathrm{M} \mathrm{NaCl}$ ) and contain Cdc37. Hsp90 inhibitors that bind to its C-terminal domain (C-terminal inhibitor), like novobiocin (4), appear to block the interaction of Hsp90 with most co-chaperones, as well as protein clients. Celastrol (2), which is thought to bind to the $\mathrm{N}$-terminal domain of Hsp90 at a site distinct from the ATP binding pocket, inhibits Hsp90 in a manner distinct from geldanamycin (3) and novobiocin (4). ${ }^{25}$

HRI is a heme-regulated eIF $2 \alpha$ kinase that requires Hsp90, Hsp70, and Cdc37 for its maturation and activation under heme-deficient conditions. ${ }^{27-29}$ To further characterize the mechanism of action of gambogic acid (1), the effects of $\mathbf{1}$ on the binding of Hsp90 chaperone components to HRI were compared to the effects of the $\mathrm{N}$-terminal inhibitors geldanamycin (3) and celastrol (2), molybdate, and the C-terminal inhibitors novobiocin (4) and coumermycin A1 (5). ${ }^{25}$ The nonactivatable K199R mutant of HRI was used in these experiments, as it is unable to mature and interacts constitutively with Hsp90. In these experiments, the immunoadsorbed samples were washed with low ionic strength buffer, as it distinguishes between the mechanism of action of geldanamycin (3) and celastrol (2), which bind to different sites in the Hsp90s N-terminal domain. In agreement with results reported previously, ${ }^{30-32}$ (1) geldanamycin (3) caused the accumulation of Hsp90 in complexes with HRI/ K199R that contained increased amounts of the intermediate cochaperone component Hsp70 compared to the DMSO control, but lacked Cdc37 (Figure 3A, lane 3); (2) molybdate stabilized the binding of Hsp90 and Cdc37 to HRI/K199R, while not affecting the interaction of Hsp70 with HRI/K199R (lane 4); and (3) novobiocin (4) disrupted the interaction of Hsp90, Hsp70, and Cdc37 with HRI/K199R (lane 6), as did the bivalent novobiocin-related compound coumermycin A1 (5) (lane 7). Under similar conditions, celastrol (2) disrupted the binding of Hsp90, Hsp70, and Cdc37 to HRI (Figure 3A, lane 5), as did gambogic acid (1) (lane 8). These results indicate that $\mathbf{1}$ affected the interaction of Hsp90 chaperone components with $\mathrm{HRI} / \mathrm{K} 199 \mathrm{R}$ in a manner distinct from geldanamycin (3) and molybdate.

Subsequently, the effects of $\mathbf{1}$ on HRI activation were examined upon incubation in heme-deficient lysate. Hsp90-dependent maturation and activation of HRI are accompanied by a change in its electrophoretic mobility to a more slowly migrating species (Figure 3B). After 45 min of incubation of HRI in heme-deficient lysate, approximately half of the $\left[{ }^{35} \mathrm{~S}\right] \mathrm{HRI}$ had a slower electrophoretic mobility upon SDS-PAGE analysis compared to HRI incubated in heme-replete lysate (lane 2 versus 1). Gambogic acid (1) inhibited the maturation/activation of HRI in hemedeficient lysate to an extent similar to the known Hsp90 inhibitors geldanamycin (3), celastrol (2), and molybdate. The C-terminal Hsp90 inhibitors novobiocin (4) and coumermycin A1 (5) also inhibited the maturation/activation of HRI, but it was also apparent that incubation with these compounds reduced the quantity of $\left[{ }^{35} \mathrm{~S}\right] \mathrm{HRI}$ that was present in the samples compared to the other Hsp90 inhibitors.

To determine whether the decreased recovery of $\left[{ }^{35} \mathrm{~S}\right] \mathrm{HRI}$ was a hallmark of C-terminal Hsp90 inhibitors inducing the breakdown of HRI or whether it was simply due to less $\left[{ }^{35} \mathrm{~S}\right] \mathrm{HRI}$ being synthesized, aliquots were taken from each sample after protein synthesis had ceased, prior to the beginning of the maturational incubation in the presence of the compounds. Autoradiography of aliquots taken from samples prior to their maturational incubation indicated that an equivalent amount of $\left[{ }^{35} \mathrm{~S}\right]$ HRI was present in each sample (Figure 3C, 0 min: upper panel). Incubation of $\left[{ }^{35} \mathrm{~S}\right] \mathrm{HRI}$ in the presence of novobiocin (4) or coumermycin A1 (5) resulted in a $90 \%$ and $80 \%$ loss of the $\left[{ }^{35} \mathrm{~S}\right] \mathrm{HRI}$, respectively (Figure 3C, lanes 6 and 7). However, significantly less $\left[{ }^{35} \mathrm{~S}\right] \mathrm{HRI}$ was lost upon incubation of samples in the presence of geldanamycin (3) (0\%), molybdate (10\%), celastrol (2) (40\%), or 1 (10\%) compared to the DMSO control. A similar effect of novobiocin (4) and coumermycin Al (5) has been observed on the stability of Akt generated by TnT in reticulocyte lysate (not shown). Thus, the effect of Hsp90 
A. Co-immunoadsorption

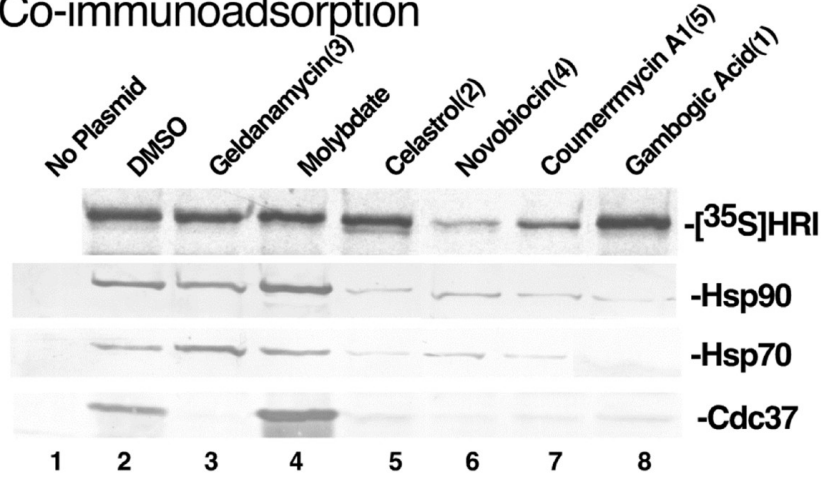

B. HRI Maturation

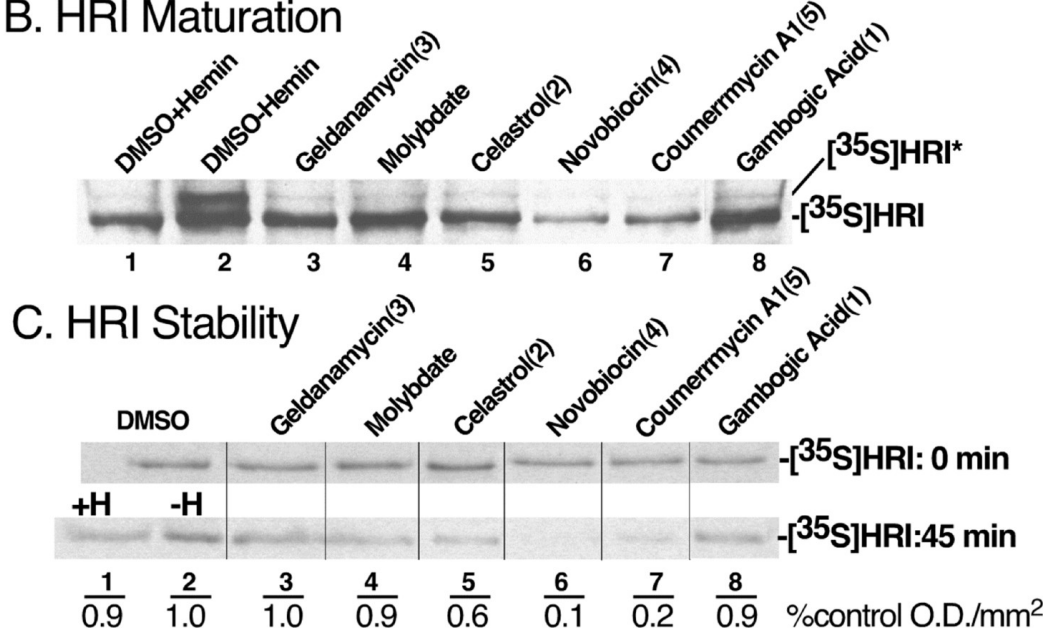

Figure 3. Effect of Hsp90 inhibitors on the interaction of Hsp90 and its co-chaperones with HRI (A), on HRI's Hsp90-dependent maturation (B), and on HRI stability (C). (A) $\left[{ }^{35} \mathrm{~S}\right]$ His-tagged HRI/K199R was synthesized by TnT in reticulocyte lysate as described in the Experimental Section. After $10 \mathrm{~min}$, DMSO (4\% v/v, lanes 1 and 2), geldanamycin $(3,80 \mu \mathrm{M}$, lane 3), sodium molybdate (20 mM, lane 4), celastrol (2, $100 \mu \mathrm{M}$, lane 5), novobiocin (4, $4.0 \mathrm{mM}$, lane 6), coumermycin A1 (5, $400 \mu \mathrm{M}$, lane 7), or gambogic acid (1,50 $\mu \mathrm{M}$, lane 8) was added, followed by an additional 40 min of incubation. $\left[{ }^{35} \mathrm{~S}\right]$ His-tagged HRI/K199R was then immunoadsorbed with anti-His antibodies, and samples were analyzed for coadsorbing Hsp90, Hsp70, and Cdc37 by SDS-PAGE and Western blotting. Lane 1: TnT lysate containing no plasmid as the control for nonspecific binding. Top panel: autoradiogram of immunoadsorbed $\left[{ }^{35} \mathrm{~S}\right] \mathrm{HRI} / \mathrm{K} 199 \mathrm{R}$. (B) $\left[{ }^{35} \mathrm{~S}\right]$ His-tagged HRI was synthesized in reticulocyte lysate as described in the Experimental Section. After $20 \mathrm{~min}$, DMSO (4\% v/v, lanes 1, 2, and 3), geldanamycin (3, $80 \mu \mathrm{M}$, lane 4), sodium molybdate (20 mM, lane 5), celastrol (2, $100 \mu \mathrm{M}$, lane 6), novobiocin (4, 4.0 mM, lane 7), coumermycin A1 (5, $400 \mu \mathrm{M}$, lane 8), or 1 (50 $\mu \mathrm{M}$, lane 9) was added, followed by an additional 10 min of incubation. An aliquot of the TnT lysate was then transferred to hemin-supplemented (20 $\mu \mathrm{M}$, lane 2) or heme-deficient (lanes 1 and 3-12) lysate containing an equivalent concentration of each addition, followed by an additional $45 \mathrm{~min}$ of incubation. The samples were then analyzed for HRI maturation by SDS-PAGE and autoradiography as described in the Experimental Section. Lane 1: TnT lysate containing no plasmid. $\left.{ }^{35} \mathrm{~S}\right] \mathrm{HRI} \mathrm{I}^{*}: \mathrm{mature}$, active HRI. (C) $\left[{ }^{35} \mathrm{~S}\right]$ His-tagged HRI was synthesized in reticulocyte lysate and treated with DMSO or Hsp90 inhibitors as described above. Aliquots of each reaction were taken prior to (upper panel, $0 \mathrm{~min}$ ) and $45 \mathrm{~min}$ after (lower panel, $45 \mathrm{~min}$ ) dilution into and incubation in heme-supplemented (lane 1) or heme-deficient (lanes $2-8$ ) lysate. The band intensities in the lower panel were quantified by scanning densitometry, and values below the lane numbers are given as $\% \mathrm{OD} / \mathrm{mm}^{2}$ of minus heme control.

inhibition on the stability of nascent kinases in reticulocyte lysate appears to be a property that further distinguishes inhibitors of Hsp90 that bind to its C-terminal domain from inhibitors that bind to its N-terminal domain. ${ }^{25,26}$ The absence of an effect of 1 on HRI stability distinguishes its mechanism of action from that of C-terminal inhibitors. Previously, celastrol (2) was demonstrated to inhibit Hsp90 by a mechanism that was distinct from $\mathrm{N}$-terminal inhibitors that bind to the Hsp90s ATP binding pocket, such as geldanamycin (3). ${ }^{25}$ The results presented here indicate that the mechanism of action of $\mathbf{1}$ is similar to that of celastrol (2) and distinct from that of 3.

Surface Plasmon Resonance (SPR) Analysis of the Binding of Gambogic Acid (1) to the N-Terminal Domain of Hsp90. To determine whether $\mathbf{1}$ is indeed interacting with the Hsp90s $\mathrm{N}$-terminal domain, we cloned the $\mathrm{N}$-terminal domain of
Hsp90 $\alpha$ (Hsp90NT; amino acids 1-241) with a seven-aminoacid extension at its C-terminus containing a C-terminal Cys residue. Recombinant Hsp90NT was biotinylated and immobilized onto a neutravidin sensor chip for analysis of the binding of 1 by SPR. Full-length Hsp90 and the C-terminal domain of Hsp90 (Hsp90CT, amino acids 531-732) were immobilized on sensor chips that were used as positive and negative controls, respectively. Gambogic acid (1) was observed to bind specifically to full-length Hsp90 (Figure 4A) and Hsp90NT (Figure 4B), but not to Hsp90CT (data not shown). Analysis of binding and dissociation kinetics indicated that 1 binds to both Hsp90 and Hsp90NT with similar association constants $\left(k_{\mathrm{a}}\right)$, dissociation constants $\left(k_{\mathrm{d}}\right)$, and calculated $K_{\mathrm{d}}$ values $(9.8$ versus $7.6 \mu \mathrm{M}$, respectively, Table 1$)$. Compound 1 was observed also to bind Hsp90NT in the presence of $20 \mu \mathrm{M}$ geldanamycin in the analyte 

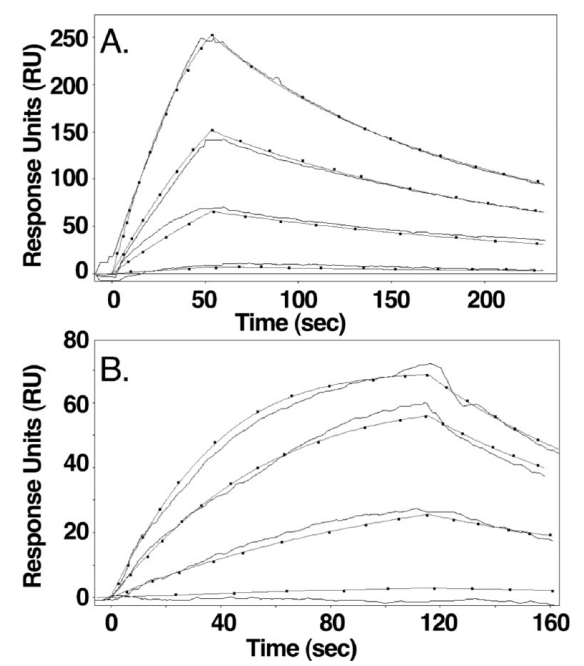

Figure 4. SPR analysis of the interaction of gambogic acid (1) with (A) full-length Hsp90 and (B) the N-terminal domain of Hsp90. (A) Injection of $1.0,10,25$, and $50 \mu \mathrm{M} 1$ over a SPR chip containing bound full-length Hsp90. (B) Injection of $0.5,5,15$, and $25 \mu \mathrm{M} 1$ over a SPR chip containing bound Hsp90NT. Black line: sensorgram of binding and dissociation; gray line with dots: curve fit.

Table 1. Constants for the Binding of Gambogic Acid (1) to Hsp90

\begin{tabular}{lccc}
\multicolumn{1}{c}{ protein } & $k_{\mathrm{a}}\left(\mathrm{M}^{-1} \mathrm{~s}^{-1}\right)$ & $k_{\mathrm{d}}\left(\mathrm{s}^{-1}\right)$ & $K_{\mathrm{D}}(\mu \mathrm{M})$ \\
full-length Hsp90 & $1.16(8) \times 10^{3}$ & $0.0113(4)$ & $9.8(2)$ \\
Hsp90NT & $1.47(6) \times 10^{3}$ & $0.01122(7)$ & $7.6(3)$ \\
Hsp90NT $(+20 \mu \mathrm{M} \mathrm{GA})$ & $1.62(8) \times 10^{3}$ & $0.0114(7)$ & $7.0(4)$ \\
Hsp90CT & \multicolumn{3}{c}{ no binding } \\
\hline
\end{tabular}

buffer, with geldanamycin (3) having no significant effect on the measured $k_{\mathrm{a}}$ and $k_{\mathrm{d}}$ or the calculated $K_{\mathrm{d}}($ Table 1$)$. Thus, the data indicate that gambogic acid binds to the $\mathrm{N}$-terminal domain of Hsp90, and, like celastrol, ${ }^{11}$ it binds to a site distinct from the ATP binding pocket. In addition, the $K_{\mathrm{d}}$ for the binding of $\mathbf{1}$ to Hsp90 correlated well with the $\mathrm{IC}_{50}$ values for gambogic acid (1)-induced inhibition of other Hsp90-dependent processes, further supporting Hsp90 as one of the physiological targets of this compound.

Virtual Docking of Gambogic Acid (1) to Hsp90NT. We approached the problem of identifying the putative binding site of gambogic acid (1) in the N-terminal domain of Hsp90 using the "blind" docking method, ${ }^{33}$ in which the entire Hsp90 $\mathrm{N}$-terminal domain, not just the geldanamycin-binding site, was used to search for the lowest possible binding energy for $\mathbf{1}$. The apo- and geldanamycin-bound Hsp90NT crystal structure, PDB ID 1YET, along with the open or unbound crystal structure of the Hsp90NT, PDB ID 1YES, were used for docking of $\mathbf{1}$ to determine if it would compete for the geldanamycin-binding site or bind elsewhere. These two structures share a $0.25 \AA$ rmsd of the $C \alpha$ backbone spanning the entire 221 residues of the crystal structures with the majority of conformational changes occurring in residues $105-112 .{ }^{34}$ In all three docking experiments, $\mathbf{1}$ preferentially bound to the cleft created by $\alpha$-helix 9 (H9) and $\beta$-sheet strand 8 (S8), residues 200-222 (Figure 5A) that are composed of both hydrophobic and positively charged elements (Figure 5B). The calculated average binding affinities for $\mathbf{1}$ for

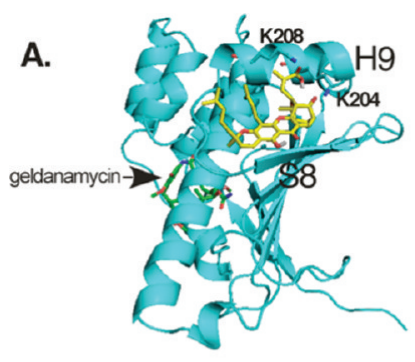

B.
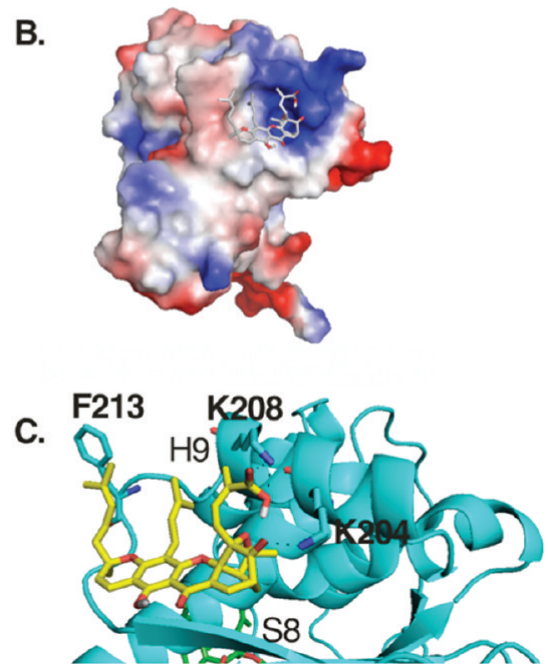

Figure 5. Models of gambogic acid (1) docked to geldanamycin-bound Hsp90NT. (A) Ribbon diagram of Hsp90NT with the carbons of $\mathbf{1}$ and geldanamycin shown in yellow and green, respectively. (B) Electrostatic surface potential of Hsp90NT shown in the same orientation as in A. (C) Close-up showing the salt bridge and $\mathrm{H}$-bond formed between $\mathrm{K} 208$ and K204 with 1.

this region were $-9.44,-9.50$, and $-9.37 \mathrm{kcal} / \mathrm{mol}$ for the apoand geldanamycin-bound and open structures, respectively. Docking results predict that aliphatic hydrophobic interactions would occur between the gambogic acid substituent arms and the aliphatic side chains of $\mathrm{H} 9$ and Phe213 in the intervening loop, while the xanthenone moiety would lie on the $C \alpha$ backbone of S8, making a potential $\pi$ interaction with the carbonyl and amide bonds (Figure 5A). In addition, there is a potential salt bridge between Lys208 of $\mathrm{H} 9$ and both oxygens of the carboxylic acid moiety of $\mathbf{1}$, and a hydrogen bond between Lys 204 of $\mathrm{H} 9$ and the carbonyl moiety of the cyclohexene ring (Figure 5C). The regions of $\mathrm{H} 9$ and $\mathrm{S} 8$ undergo minor conformational changes with a $0.15 \AA$ rmsd of the $\mathrm{C} \alpha$ residues between residues $200-222$.

The docking results are consistent with gambogic acid (1) being a noncompetitive inhibitor of the Hsp90 N-terminal domain. The region of H9 and S8 of the HSP90 N-terminal domain undergoes little change between the geldanamycinbound structure and the open conformation structure with a $0.15 \AA$ rmsd of the C $\alpha$ backbone of residues 200-222. It should be noted that this site is distinct from that previously proposed for the binding of celastrol (2) to the Hsp90s N-terminal domain. $^{12}$

Recently, the combination of gambogic acid (1) and celastrol (2) was reported to have a synergistic antitumor effect, ${ }^{35}$ an observation that supports the notion that the two compounds interact with different sites within the $\mathrm{N}$-terminal domain of 
Hsp90. The question of whether combinations of Hsp90 inhibitors that act by different mechanisms will demonstrate similar synergistic effects on cancer cell growth has yet to be explored, but the synergy exhibited by $\mathbf{1}$ and $\mathbf{2}$ suggests that such studies would be productive. For example, the ability of C-terminal inhibitors, such as novobiocin, to block the activation of HSF $1^{36}$ may potentiate the cytoxicity of N-terminal inhibitors of Hsp90, which induce heat shock protein expression.

Conclusion. The data presented above firmly establish gambogic acid (1) as an inhibitor of $\mathrm{Hsp} 90$ and provide evidence of a new site that can be targeted for the development of improved Hsp90 inhibitors. Previous work with 1 has indicated that it reduces the activity or the levels of expression of well-known Hsp90-dependent clients (see: http://www.picard.ch/downloads/Hsp90interactors.pdf and http://www.picard.ch/downloads/Hsp90facts.pdf) that are involved in the regulation of cell immortality (telomerase), ${ }^{37,38}$ cell growth (Akt, cSrc, Cdk2, and Cdk4), ${ }^{39-41}$ apoptosis (Bcl-2, Bcl-xL, and survivin), ${ }^{42-44}$ angiogenesis (VEGFR), ${ }^{40,41}$ and metastasis (MMP2 and MMP9). ${ }^{2,45}$ Changes in levels of expression of proteins (Bcl2, Bcl-xL, survivin, and telomerase) appeared to occur primarily at the level of transcription rather than through destabilization of the protein, as mRNA levels coding for the protein were decreased. These changes, however, can still be accounted for by a mechanism invoking gambogic acid-induced inhibition of Hsp90, as NF- $\kappa$ B regulates the expression of $\mathrm{Bcl}-2, \mathrm{Bcl}-\mathrm{xL}$, and survivin, as well as VEGF, ${ }^{17,22}$ and NF- $\kappa \mathrm{B}$ activation is well known to be regulated by Hsp90. ${ }^{46}$ This notion is supported by the observations that the Hsp90 inhibitor geldanamycin (3) inhibits the expression of $\mathrm{Bcl}-2$ and $\mathrm{Bcl}-\mathrm{xL}$ by suppressing the activation of NF- $\kappa \mathrm{B}^{47}$ and has the same effects as gambogic acid on VEGFR signaling. ${ }^{48,49}$ Furthermore, telomerase expression is regulated by c-Myc, and $\mathrm{Hsp} 90$ inhibition has recently been demonstrated to destabilize c-Myc, ${ }^{50}$ in addition to blocking the interaction of Hsp90 with the telomerase promoter, which suppresses telomerase expression. ${ }^{51}$ While this article was in preparation, gambogic acid (1) was confirmed to block NF- $\kappa \mathrm{B}$ activation through its ability to inhibit Hsp90. ${ }^{52}$ Thus, many of the physiological effects of $\mathbf{1}$ on cultured cells reported previously in the literature can be accounted for, in part, by its ability to inhibit Hsp90 function.

\section{EXPERIMENTAL SECTION}

Screen for Inhibitors of Hsp90-Dependent Luciferase Refolding. Natural product libraries from Microsource and Biomol were screened as previously described for inhibitors of Hsp90-dependent refolding of thermally denatured firefly luciferase. ${ }^{53}$ Positive hits were then screened against native luciferase to eliminate false positives that were direct inhibitors of luciferase. Subsequently, celastrol (2, CalBiochem $219465, \geq 95 \%$ by TLC) and gambogic acid (1, Biomol BML-AP305, 98\% by TLC) were titrated into reticulocyte lysate containing thermally denatured luciferase to determine the concentration of test compound required to inhibit luciferase refolding by $50 \%$ $\left(\mathrm{IC}_{50}\right)$ compared to the DMSO control, as previously described. ${ }^{54}$ Reactions were carried out in triplicate at room temperature in 96-well microtiter plates, and experiments were repeated at least twice, with relative light unit (RLU) production measured using a $\mathrm{L}_{\mathrm{Max}} \mathrm{II}$ (Molecular Devices) microplate reader and a $10 \mathrm{~s}$ integration time. ${ }^{54}$

Effect of Gambogic Acid (1) on Cell Proliferation. MCF-7 and HeLa cells were grown in Gibco modified essential medium, supplemented with nonessential amino acids, $2 \mathrm{mM}$ glutamine, and
$10 \%$ fetal bovine serum. SkBr3 cells were maintained in DMEM/F12 (1:1) medium (Gibco) with L-glutamine supplemented with streptomycin $(500 \mu \mathrm{g} / \mathrm{mL})$, penicillin (100 units $/ \mathrm{mL}$ ), and $10 \%$ fetal bovine serum. Cells were grown to confluence in a humidified atmosphere $\left(37^{\circ} \mathrm{C}, 5 \% \mathrm{CO}_{2}\right)$. Cells were seeded at 2000 cells per well in a clear $96-$ well plate, the medium volume was brought to $100 \mu \mathrm{L}$, and the cells were allowed to attach overnight. The next day, varying concentrations of compound or $1 \%$ DMSO vehicle control was added to the wells. Cells were then incubated at $37{ }^{\circ} \mathrm{C}$ for $72 \mathrm{~h}$. Cell viability was determined using the Promega Cell Titer 96 Aqueous One Solution cell proliferation assay. After incubation with compounds, $20 \mu \mathrm{L}$ of the assay substrate solution was added to the wells, and the plate was incubated at $37^{\circ} \mathrm{C}$ for an additional $1 \mathrm{~h}$. Absorbance at $490 \mathrm{~nm}$ was then read on a Molecular Devices Versamax plate reader, and values were expressed as percent of absorbance from cells incubated in DMSO alone.

Effect of Gambogic Acid (1) on the Interaction of Hsp90 and Its Co-chaperones with Heme-Regulated elF2 $\alpha$ Kinase (HRI). His-tagged kinase-dead HRI/K199R was synthesized by coupled transcription-translation ( $\mathrm{TnT}$ ) in reticulocyte lysate in the presence of $\left[{ }^{35} \mathrm{~S}\right]$ methionine. ${ }^{55,56}$ After $20 \mathrm{~min}$ of synthesis, compounds or an equivalent volume of DMSO or water was added at the concentrations indicated in the figure legend, and synthesis was continued for an additional $10 \mathrm{~min}$. Subsequently, aliquots were taken for immunoadsorption of His-tagged HRI with anti-His-tag antibodies bound to antimouse-IgG agarose resin. ${ }^{55,56}$ Agarose resins containing bound anti-Histag antibody were washed four times with $10 \mathrm{mM}$ PIPES $(\mathrm{pH}=7.2)$ plus $100 \mathrm{mM} \mathrm{NaCl}$ and analyzed by SDS-PAGE and Western blotting for coadsorbed Hsp90, Hsp70, and Cdc37, as previously described. ${ }^{55,56}$ Lysate lacking plasmid encoding His-tagged HRI was used as the control for nonspecific binding.

Effect of Gambogic Acid (1) on Hsp90-Dependent HRI Maturation/Activation. $\left[{ }^{35}\right.$ S] His-tagged wild-type HRI or K199R mutant was generated by $\mathrm{TnT}$ in reticulocyte lysate, as described above. After $30 \mathrm{~min}$, samples were diluted into 7 volumes of hemin-supplemented or heme-deficient lysate containing DMSO, water, or test compound at the same concentration present in the TnT lysate and incubated for an additional $45 \mathrm{~min}^{55,56}$ The His-tagged HRI was adsorbed from samples by the addition of NTA-Ni resin on ice for $1 \mathrm{~h}$. Resins were washed and eluted by boiling in SDS-sample buffer, and samples were analyzed by SDS-PAGE and autoradiography after electrotransfer to PVDF membrane to detect a shift in the electrophoretic mobility of HRI, which is dependent upon its Hsp90-dependent maturation/activation. ${ }^{55,56}$

Gambogic Acid (1)-Induced Depletion of Hsp90-Dependent Proteins from Cultured MCF7 and SKBr3 Breast Cancer and HeLa Cells. MCF-7, SkBr3, and HeLa cells were grown to confluence as described above, seeded in culture dishes $\left(1 \times 10^{6} /\right.$ dish $)$, and allowed to attach overnight. Gambogic acid (1) was added at the concentrations indicated in Figure 1, and the cells were incubated for an additional $36 \mathrm{~h}$. Cells were harvested and analyzed for Hsp90 client protein degradation (Her2, Raf-1, and Akt) and heat shock protein induction (Hsp90 and Hsp70), as described previously. ${ }^{57}$ For comparison, cells were incubated with DMSO (1\%) or geldanamycin $(3,500 \mathrm{nM})$, and extracts $(10 \mu \mathrm{g}$ of protein) were Western blotted for the indicated proteins with actin used as the loading control. One microgram of $\mathrm{SkBr} 3$ extracts was used for the Western blot for Her2.

Surface Plasmon Resonance Spectroscopy of Gambogic Acid (1) Binding to Hsp90. Insect Sf9 cells overexpressing human Hsp90 $\beta$ were cultured and harvested by the Baculovirus/Monoclonal Antibody Core Facility at Baylor College of Medicine. Hsp90 $\beta$ was extracted and purified ( $>98 \%$ pure) as described previously, ${ }^{58,59}$ but without the initial DEAE-cellulose chromatography step. Bacterial E. coli DE-3 Star cells carrying plasmids for the expression of either the 
N-terminal (Hsp90NT: amino acids 1-241 with a C-terminal -GELRSGC tail) or C-terminal (Hsp90CT: amino acids 531-732) domains of Hsp90 were cultured in LB medium and induced with IPTG. Recombinant Hsp90NT and Hsp90CT were purified using a NiNTA column. Following elution of the protein from the NiNTA column, the $\mathrm{N}$-terminal His-tag was cleaved using TEV protease (Invitrogen), followed by size-exclusion chromatography on Superdex 200. The Hsp90-containing fractions were pooled, concentrated, dialyzed against $10 \mathrm{mM}$ Hepes ( $\mathrm{pH} 7.5$ ) containing $150 \mathrm{mM} \mathrm{NaCl}$ and $10 \%$ glycerol, and stored at $-80{ }^{\circ} \mathrm{C}$ until use. Prior to the use of the Hsp90NT or Hsp90CT, each protein was reacted according to the manufacture's recommended protocol with the EZ-Link Maleimide-PEG2-Biotin (Thermo Scientific) then buffer-exchanged into fresh $10 \mathrm{mM}$ Hepes ( $\mathrm{pH} 7.5$ ) containing $150 \mathrm{mM} \mathrm{NaCl}$ to eliminate free biotin entities.

The surface of a SSO $1 \mathrm{CO}_{2} \mathrm{H}$ SPR sensor chip mounted in a SensiQ SPR instrument (ICX Nomadics) was activated by treatment with $N^{\prime}-3-$ dimethylaminopropyl- $N^{\prime}$-ethylcarbodiimide hydrochloride and $N$-hydroxysuccinimide for preferential cross-linking of full-length Hsp90s $\mathrm{N}$-terminus to the surface. For immobilization of Hsp90, $250 \mu \mathrm{L}$ of Hsp90 $(6.2 \mathrm{mg} / \mathrm{mL})$ in $10 \mathrm{mM}$ Hepes buffer $(\mathrm{pH} 7.4)$ containing $150 \mathrm{mM} \mathrm{NaCl}$ was injected at a flow rate of $10 \mu \mathrm{L} / \mathrm{min}$, resulting in 2000 response units of protein captured on the experimental surface of the chip. Then, $1 \mathrm{M}$ ethanolamine ( $\mathrm{pH} 8$ ) was used to quench the remaining activated groups, and the surface washed with buffer containing $10 \mathrm{mM}$ PIPES pH 7.4, $300 \mathrm{mM} \mathrm{NaCl}$, and 2\% DMSO. The surface of a SSO3 BioCap SPR sensor chip was mounted in a SensiQ SPR instrument (ICX Nomadics), and either the biotinylated Hsp90NT $(7.0 \mathrm{mg} / \mathrm{mL})$ or Hsp90CT $(6.8 \mathrm{mg} / \mathrm{mL})$ was discrete-injected over the experimental channel at a flow rate of $10 \mu \mathrm{L} / \mathrm{min}$, resulting in capture of Hsp90NT at 1400 response units and $\mathrm{Hsp} 90 \mathrm{CT}$ at 1250 response units of protein on the experimental surface of the chip. The chips were then washed with assay buffer prior to experimental analysis.

Gambogic acid (1) was diluted in assay buffer containing $10 \mathrm{mM}$ PIPES pH 7.4, $300 \mathrm{mM} \mathrm{NaCl}$, and 2\% DMSO and injected over the surface of each derivatized chip at a flow rate of $15 \mu \mathrm{L} / \mathrm{min}$ at $25^{\circ} \mathrm{C}$ at the indicated concentrations. Additionally, for competition studies 1 was diluted in assay buffer containing $20 \mu \mathrm{M}$ geldanamycin (3). All measurements were done in triplicate. SPR binding curves were analyzed using QDAT software (ICX Nomadics) to calculate the $k_{a}, k_{\mathrm{d}}$ and $K_{\mathrm{D}}$ data.

Docking Studies of Geldanamycin (3) and Gambogic Acid (1) with the N-Terminal Domain of Hsp90. In silico docking of geldanamycin and gambogic acid with the $3 \mathrm{D}$ coordinates of the X-ray crystal structures of the N-terminal domain of HSP90 with bound 3 and in the open conformation, PDB IDs 1YET and 1YES, respectively, was accomplished using the AutoDock program ${ }^{60}$ downloaded from the Molecular Graphics Laboratory of the Scripps Research Institute. The AutoDock program was chosen because it uses a genetic algorithm to generate the poses of the ligand inside a known or predicted binding site utilizing the Lamarckian version of the genetic algorithm where the changes in conformations adopted by molecules after in situ optimization are used as subsequent poses for the offspring.

In the docking experiments carried out, water was removed from the 3D X-ray coordinates while Gasteiger charges were placed on the X-ray structures of the N-terminal domain of HSP90 along with geldanamycin (3) and $\mathbf{1}$ using tools from the AutoDock suite. A grid box centered on the N-terminal HSP90 domain with definitions of $126 \times 126 \times 126$ points and $0.4 \AA$ spacing was chosen for ligand docking experiments. The docking parameters consisted of setting the population size to 300 , the number of generations to 27000 , and the number of evaluations to 20000000 , while the number of docking runs was set to 50 with a cutoff of $1 \AA$ for the root-mean-square tolerance for the grouping of each docking run.

While the binding mode of geldanamycin with Hsp90 has been determined through X-ray crystallography, ${ }^{34}$ the binding mode of $\mathbf{1}$ with
Hsp90 has yet to be determined through either NMR spectroscopy or $\mathrm{X}$-ray crystallography. The docking of geldanamycin to the apo X-ray structure of Hsp90NT, PDB ID 1YET, was used as a control to test and validate the docking parameters used. As expected, geldanamycin (3) docked to the binding site identified in the crystal structure with an average binding energy of $-9.65 \mathrm{kcal} / \mathrm{mol}$ and a 1 A average root-meansquare deviation from the reference structure.

\section{AUTHOR INFORMATION}

\section{Corresponding Author}

*Tel: (405)744-6200. Fax: (405)744-7799. E-mail: robert.matts@ okstate.edu.

\section{ACKNOWLEDGMENT}

The authors gratefully acknowledge support of this project by NIH (R01 CA125392) and the Oklahoma Agricultural Experiment Station (Project No. 1975).

\section{REFERENCES}

(1) Pearl, L. H.; Prodromou, C.; Workman, P. Biochem. J. 2008, 410, 439-453.

(2) Pratt, W. B.; Toft, D. O. Exp. Biol. Med. (Maywood) 2003, 228, 111-133.

(3) Neckers, L. J. Biosci. 2007, 32, 517-530.

(4) Hanahan, D.; Weinberg, R. A. Cell 2000, 100, 57-70.

(5) Holzbeierlein, J. M.; Windsperger, A.; Vielhauer, G. Curr. Oncol. Rep. 2010, 12, 95-101.

(6) Bishop, S. C.; Burlison, J. A.; Blagg, B. S. Curr. Cancer Drug Targets 2007, 7, 369-388.

(7) Donnelly, A.; Blagg, B. S. Curr. Med. Chem. 2008, 15, 2702-2717.

(8) Li, Y.; Zhang, T.; Jiang, Y.; Lee, H. F.; Schwartz, S. J.; Sun, D. Mol. Pharmaceutics 2009, 6, 1152-1159.

(9) Yin, Z.; Henry, E. C.; Gasiewicz, T. A. Biochemistry 2009, $48,336-345$.

(10) Brandt, G. E.; Schmidt, M. D.; Prisinzano, T. E.; Blagg, B. S. J. Med. Chem. 2008, 51, 6495-6502.

(11) Hieronymus, H.; Lamb, J.; Ross, K. N.; Peng, X. P.; Clement, C.; Rodina, A.; Nieto, M.; Du, J.; Stegmaier, K.; Raj, S. M.; Maloney, K. N.; Clardy, J.; Hahn, W. C.; Chiosis, G.; Golub, T. R. Cancer Cell 2006, 10, 321-330.

(12) Zhang, T.; Hamza, A.; Cao, X.; Wang, B.; Yu, S.; Zhan, C. G.; Sun, D. Mol. Cancer Ther. 2008, 7, 162-170.

(13) Oh, S. H.; Woo, J. K.; Yazici, Y. D.; Myers, J. N.; Kim, W. Y.; Jin, Q.; Hong, S. S.; Park, H. J.; Suh, Y. G.; Kim, K. W.; Hong, W. K.; Lee, H. Y. J. Natl. Cancer Inst. 2007, 99, 949-961.

(14) Porter, J. R.; Fritz, C. C.; Depew, K. M. Curr. Opin. Chem. Biol. 2010, 14, 412-420.

(15) Kim, Y. S.; Alarcon, S. V.; Lee, S.; Lee, M. J.; Giaccone, G.; Neckers, L.; Trepel, J. B. Curr. Top. Med. Chem. 2009, 9, 1479-1492.

(16) Aggarwal, B. B.; Kunnumakkara, A. B.; Harikumar, K. B.; Tharakan, S. T.; Sung, B.; Anand, P. Planta Med. 2008, 74, 1560-1569.

(17) Gupta, S. C.; Kim, J. H.; Prasad, S.; Aggarwal, B. B. Cancer Metastasis Rev. 2010, 29, 405-434.

(18) Han, Q. B.; Xu, H. X. Curr. Med. Chem. 2009, 16, 3775-3796.

(19) Kamal, A.; Thao, L.; Sensintaffar, J.; Zhang, L.; Boehm, M. F.; Fritz, L. C.; Burrows, F. J. Nature 2003, 425, 407-410.

(20) Yang, Y.; Yang, L.; You, Q. D.; Nie, F. F.; Gu, H. Y.; Zhao, L.; Wang, X. T.; Guo, Q. L. Cancer Lett. 2007, 256, 259-266.

(21) Zhao, L.; Guo, Q. L.; You, Q. D.; Wu, Z. Q.; Gu, H. Y. Biol. Pharm. Bull. 2004, 27, 998-1003.

(22) Pandey, M. K.; Sung, B.; Ahn, K. S.; Kunnumakkara, A. B.; Chaturvedi, M. M.; Aggarwal, B. B. Blood 2007, 110, 3517-3525.

(23) Ortiz-Sanchez, E.; Daniels, T. R.; Helguera, G.; Martinez-Maza, O.; Bonavida, B.; Penichet, M. L. Leukemia 2009, 23, 59-70. 
(24) Ren, Y.; Yuan, C.; Chai, H. B.; Ding, Y.; Li, X. C.; Ferreira, D.; Kinghorn, A. D. J. Nat. Prod. 2011, 74, 460-463.

(25) Matts, R. L.; Brandt, G. E.; Lu, Y.; Dixit, A.; Mollapour, M.; Wang, S.; Donnelly, A. C.; Neckers, L.; Verkhivker, G.; Blagg, B. S. Bioorg. Med. Chem. 2011, 19, 684-692.

(26) Matts, R. L.; Manjarrez, J. R. Curr. Top. Med. Chem. 2009, 9, 1462-1478.

(27) Shao, J.; Gramatikakis, N.; Scroggins, B.; Uma, S.; Huang, W.; Chen, J.-J.; Hartson, S. D.; Matts, R. L. J. Biol. Chem. 2001, 276, 206-214.

(28) Uma, S.; Hartson, S. D.; Chen, J.-J.; Matts, R. L. J. Biol. Chem. 1997, 272, 11648-11656.

(29) Uma, S.; Thulasiraman, V.; Matts, R. L. Mol. Cell. Biol. 1999, 19, 5861-5871.

(30) Hartson, S. D.; Thulasiraman, V.; Huang, W.; Whitesell, L.; Matts, R. L. Biochemistry 1999, 38, 3837-3849.

(31) Prince, T.; Matts, R. L. J. Biol. Chem. 2004, 279, 39975-39981.

(32) Scroggins, B. T.; Prince, T.; Shao, J.; Uma, S.; Huang, W.; Guo, Y.; Yun, B. G.; Hedman, K.; Matts, R. L.; Hartson, S. D. Biochemistry 2003, 42, 12550-12561.

(33) Hetenyi, C.; van der Spoel, D. Protein Sci. 2002, 11, 1729-1737.

(34) Stebbins, C. E.; Russo, A. A.; Schneider, C.; Rosen, N.; Hartl,

F. U.; Pavletich, N. P. Cell 1997, 89, 239-250.

(35) He, D.; Xu, Q.; Yan, M.; Zhang, P.; Zhou, X.; Zhang, Z.; Duan, W.; Zhong, L.; Ye, D.; Chen, W. BMC Cancer 2009, 9, 343.

(36) Conde, R.; Belak, Z. R.; Nair, M.; O’Carroll, R. F.; Ovsenek, N. Biochem. Cell Biol. 2009, 87, 845-851.

(37) Guo, Q. L.; Lin, S. S.; You, Q. D.; Gu, H. Y.; Yu, J.; Zhao, L.; Qi, Q.; Liang, F.; Tan, Z.; Wang, X. Life Sci. 2006, 78, 1238-1245.

(38) Wu, Z. Q.; Guo, Q. L.; You, Q. D.; Zhao, L.; Gu, H. Y. Biol. Pharm. Bull. 2004, 27, 1769-1774.

(39) Liu, Y.; Li, W.; Ye, C.; Lin, Y.; Cheang, T. Y.; Wang, M.; Zhang, H.; Wang, S.; Zhang, L. J. Atheroscler. Thromb. 2010, 17, 901-913.

(40) Lu, N.; Yang, Y.; You, Q. D.; Ling, Y.; Gao, Y.; Gu, H. Y.; Zhao, L.; Wang, X. T.; Guo, Q. L. Cancer Lett. 2007, 258, 80-89.

(41) Yi, T.; Yi, Z.; Cho, S. G.; Luo, J.; Pandey, M. K.; Aggarwal, B. B.; Liu, M. Cancer Res. 2008, 68, 1843-1850.

(42) Shu, W.; Chen, Y.; Li, R.; Wu, Q.; Cui, G.; Ke, W.; Chen, Z. Basic Clin. Pharmacol. Toxicol. 2008, 103, 530-537.

(43) Xu, X.; Liu, Y.; Wang, L.; He, J.; Zhang, H.; Chen, X.; Li, Y.; Yang, J.; Tao, J. Int. J. Dermatol. 2009, 48, 186-192.

(44) Wang, T.; Wei, J.; Qian, X.; Ding, Y.; Yu, L.; Liu, B. Cancer Lett. 2008, 262, 214-222.

(45) Qi, Q.; Lu, N.; Wang, X. T.; Gu, H. Y.; Yang, Y.; Liu, W.; Li, C.;

You, Q. D.; Guo, Q. L. Biochem. Cell Biol. 2008, 86, 386-395.

(46) Salminen, A.; Paimela, T.; Suuronen, T.; Kaarniranta, K. Immunol. Lett. 2008, 117, 9-15.

(47) Bai, J.; Sui, J.; Demirjian, A.; Vollmer, C. M., Jr.; Marasco, W.; Callery, M. P. Cancer Res. 2005, 65, 2344-2352.

(48) Masson-Gadais, B.; Houle, F.; Laferriere, J.; Huot, J. Cell Stress Chaperones 2003, 8, 37-52.

(49) Dias, S.; Shmelkov, S. V.; Lam, G.; Rafii, S. Blood 2002, 99, 2532-2540.

(50) Regan, P. L.; Jacobs, J.; Wang, G.; Torres, J.; Edo, R.; Friedmann, J.; Tang, X. X. Int. J. Oncol. 2011, 38, 105-112.

(51) Kim, R. H.; Kim, R.; Chen, W.; Hu, S.; Shin, K. H.; Park, N. H.; Kang, M. K. Carcinogenesis 2008, 29, 2425-2431.

(52) Zhang, L.; Yi, Y.; Chen, J.; Sun, Y.; Guo, Q.; Zheng, Z.; Song, S. Biochem. Biophys. Res. Commun. 2010, 403, 282-287.

(53) Galam, L.; Hadden, M. K.; Ma, Z.; Ye, Q. Z.; Yun, B. G.; Blagg, B. S.; Matts, R. L. Bioorg. Med. Chem. 2007, 15, 1939-1946.

(54) Thulasiraman, V.; Matts, R. L. Biochemistry 1996, 35, 1344313450

(55) Shao, J.; Irwin, A.; Hartson, S. D.; Matts, R. L. Biochemistry 2003, 42, 12577-12588.

(56) Yun, B.-G.; Huang, W.; Leach, N.; Hartson, S. D.; Matts, R. L. Biochemistry 2004, 43, 8217-8229.

(57) Yu, X. M.; Shen, G.; Neckers, L.; Blake, H.; Holzbeierlein, J.; Cronk, B.; Blagg, B. S. J. Am. Chem. Soc. 2005, 127, 12778-12779.
(58) Grenert, J. P.; Sullivan, W. P.; Fadden, P.; Haystead, T. A.; Clark, J.; Mimnaugh, E.; Krutzsch, H.; Ochel, H. J.; Schulte, T. W.; Sausville, E.; Neckers, L. M.; Toft, D. O. J. Biol. Chem. 1997, 272, 23843-23850.

(59) Owen, B. A.; Sullivan, W. P.; Felts, S. J.; Toft, D. O. J. Biol. Chem. 2002, 277, 7086-7091.

(60) Morris, G. M.; Goodsell, D. S.; Halliday, R. S.; Huey, R.; Hart, W. E.; Belew, R. K.; Olson, A. J. J. Comput. Chem. 1998, 19, 1639-1662. 